\title{
ROS1 rearrangements are uncommon in biliary tract cancers
}

\author{
FRANCESCA MAZZONI $^{1}$, PAOLO PETRENI $^{2}$, ENRICO VASILE $^{3}$, MICHELE PANEBIANCO $^{4}$, \\ ANDREA CASADEI-GARDINI ${ }^{5}$, FRANCESCA NEGRI ${ }^{6}$, ALICE LUNGHI ${ }^{7}$, SERENA PILLOZZI $^{1,8}$, \\ CATERINA VIVALDI ${ }^{3}$, ERIKA GERVASI ${ }^{4}$, GIOVANNI LUCA FRASSINETI ${ }^{5}$, LUCA MESSERINI ${ }^{9}$, \\ GENNY JOCOLLÉ ${ }^{10}$, ALESSANDRA BISAGNI ${ }^{11}$, LORENZO ANTONUZZO ${ }^{1 *}$ and GIULIO ROSSI ${ }^{12 *}$ \\ ${ }^{1}$ Medical Oncology Unit, AOU Careggi Hospital, I-50134 Florence; ${ }^{2}$ Medical Oncology Unit, \\ Alta Val d'Elsa Hospital, I-53036 Poggibonsi; ${ }^{3}$ Medical Oncology Unit, AOU Pisana Hospital, \\ I-56126 Pisa; ${ }^{4}$ AUSL-IRCSS, I-42122 Reggio Emilia; ${ }^{5}$ Department of Medical Oncology, \\ IRST IRCCS, I-47014 Meldola; ${ }^{6}$ Medical Oncology Unit, AOU Parma Hospital, I-43126 Parma; \\ ${ }^{7}$ Medical Oncology Unit, San Luca Hospital, I-55100 Lucca; ${ }^{8}$ Department of Experimental and \\ Clinical Medicine, University of Florence; ${ }^{9}$ Anatomical Pathology Unit, AOU Careggi Hospital, \\ I-50134 Florence; ${ }^{10}$ Regional Parini Hospital, I-11100 Aosta; ${ }^{11}$ Pathology Unit, ASL-IRCCS, \\ I-42122 Reggio Emilia; ${ }^{12}$ Operative Unit of Pathologic Anatomy, \\ Santa Maria delle Croci Hospital, I-48121 Ravenna, Italy
}

Received August 14, 2019; Accepted March 31, 2020

DOI: $10.3892 / 01.2020 .12179$

\begin{abstract}
Biliary tract cancers (BTCs) are a pool of diseases with poor prognosis and there is no orphan drug available. Currently, no molecular targets have been tested as druggable oncogenic drivers. C-ros oncogene 1 (ROS1) rearrangements have been previously described in various tumors, including BTCs; however, data regarding their incidence and biological significance are controversial. Therefore, a retrospective multicenter study was performed to assess the incidence of ROSI rearrangements in BTCs by means of immunohistochemistry and fluorescence in situ hybridization (FISH). The present study failed to demonstrate ROS1 expression in a multicenter series of 150 cases with BTCs and revealed that D4D6 was the most specific clone compared with other ROS1 primary antibodies, namely PA1-30318 and EPMGHR2. Notably, negative results obtained with D4D6 completely matched to data sorted out by FISH analysis, thus confirming a lack of ROSI gene rearrangements in BTCs and false positive results when PA1-30318 and EPMGHR2 clones were used. These results
\end{abstract}

Correspondence to: Dr Francesca Mazzoni, Medical Oncology Unit, AOU Careggi Hospital, L.go Brambilla 3, I-50134 Florence, Italy

E-mail: mazzonifr@aou-careggi.toscana.it

${ }^{*}$ Contributed equally

Key words: biliary tract cancers, ROS1 rearrangements, immunohistochemistry, fluorescence in situ hybridization, molecular target, biomarker suggest that ROS1 rearrangements may not be targets for molecular therapy of BTCs with specific inhibitors.

\section{Introduction}

Biliary tract cancers (BTCs) are rare tumors arising from the biliary tree epithelium, from the small peripheral hepatic ducts to the distal common bile duct. The progress on the treatment strategies for patients with BTCs has been slow in the past decades. The disease prognosis remains poor, with a modest improvement from 11 to $17 \%$ in terms of 5-year overall survival (OS) rates (1).

Complete surgical resection or liver transplantation, when feasible, are the only potentially curative treatments in the early stages of BTCs (2). In advanced stages, standard chemotherapy (CT) in combination with palliative supportive care, such as biliary drainage or stenting, is the only available therapeutic option, providing a survival advantage with a modest impact and benefit in terms of quality of life $(3,4)$. Gemcitabine plus cisplatin regimen is the current standard first-line treatment, with a median OS of less than 1 year (4). However, no standard second-line CT regimens have been established.

In recent years, whole-genome tumor profiling studies have identified a wide variety of genetic alterations, many of them considered targetable therapeutic options (HER2, $B R A F, F G F R 1-3, I D H 1 / 2, M E T$ and $M E K)(5,6)$. In addition, preliminary trials in selected populations treated with targeted therapies have demonstrated promising improvement in survival outcome (7-9). Immunotherapy is considered a revolutionary treatment method for selected patients and preliminary preclinical data have revealed encouraging results, even in BTCs (10).

C-ros oncogene 1 (ROS1), a proto-oncogene, encodes a receptor tyrosine kinase (RTK) without a known ligand. ROS1 
shares a high structural homology with the insulin receptor family and the anaplastic lymphoma kinase (ALK) (11). When ROS1 is constitutively activated by gene rearrangement, the RTK is overexpressed and is likely detected using immunohistochemistry (IHC). It has been reported that chromosomal rearrangements lead to fusion of ROS1 with several partner genes, resulting in the formation of a constitutively active fusion kinase (12). This kinase induces mitogen-activated protein kinase, signal transducer and activator of transcription 3 and phosphoinositide 3-kinase pathways, among others, subsequently promoting cellular transformation (12). These rearrangements, also evidenced by the aberrant expression of the RTK ROS1, have been detected in several types of cancer, including 1-2\% of lung adenocarcinoma cases, glioblastoma, cholangiocarcinoma (CAC) and others $(13,14)$. In lung cancer, clinical and epidemiological published trials have already described the incidence and prevalence of ROS1 as well as its predictive and prognostic role. However, there is currently a lack of consistent evidence regarding ROS1 gene rearrangements and its protein expression in other neoplasms, including BTCs (14).

It has been shown that ROS1 and ALK share significant homology within their respective tyrosine kinase (TK) domains. This finding led to the hypothesis that ALK tyrosine kinase inhibitors (TKIs) may also inhibit ROS1 expression (15). Based on promising preclinical data with different ALK TKIs, several clinical studies have been performed in ROS1-positive NSCLC patients with interesting results. For example, Shaw et al (15) demonstrated a progression free survival (PFS) of 19.2 months and a response rate of $72 \%$ in 50 ROS1-positive lung cancer patients treated with crizotinib (16-19).

In a case series of various tumors, ROS1 rearrangements were detected in 2 out of 23 patients $(8.7 \%)$ with BTCs (20). However, in a cohort of 56 Chinese CAC patients no ROS1 rearrangements were observed (21). Additionally, Graham et al (22) reported one case with ROS1 rearrangements among 100 CAC cases. Of note, the ROS1 positive case also harbored an IDHI mutation (22,23). Recently, two additional studies on Asiatic cohorts of BTCs patients reported ROSI rearrangements in 0 and $1.1 \%$, respectively $(24,25)$. The present study aimed to identify the incidence of ROS1 rearrangements in a retrospective, Italian and multicentric cohort of patients with BTCs. All cases were tested using IHC and the results from three different commercially available ROS1 primary antibodies (Abs) (clones D4D6, PA1-30318 and EPMGHR2) were compared. Positive cases were further analyzed by fluorescence in situ hybridization (FISH) to confirm the presence of ROS1 rearrangements.

\section{Materials and methods}

Study aim and design. The present multicenter, retrospective study was conducted by the Italian Clinical Oncology Research Group (GOIRC) and included eight Italian centers as follows: Azienda Ospedaliero-Universitaria Careggi, Florence; Regional Hospital Parini, Aosta; Santa Maria delle Croci Hospital, Ravenna; Santa Chiara Hospital, Pisa; Santa Maria Nuova Hospital, Reggio Emilia; IRSST, Meldola; Maggiore Hospital, Parma; and San Luca Hospital, Lucca. In the present study, 150 cases of BTCs, diagnosed between January 2012 and December 2015 using surgical specimens $(\mathrm{n}=98)$ or liver biopsy ( $n=52)$, were enrolled. All cases were eligible for inclusion in the study and sufficient material was available for IHC and FISH analyses. At the time of diagnosis patients were $\geq 8$ years old. All subjects provided written informed consent according to the Local Ethical Committees.

Histopathological samples were centrally reviewed and analyzed at the Pathology Units of the Regional Hospital Parini and Santa Maria delle Croci Hospital. The clinicopathological characteristics, including age, sex, disease stage, treatment and survival rate, were analyzed from the patients' medical records and referring physicians.

The association between ROS1 expression and survival parameters and clinicopathological characteristics, collected from the available medical records, was considered as the secondary endpoint of the study. OS was defined as the time between diagnosis and death, resulting from any cause. In particular, the secondary end point was to evaluate the potential prognostic role of ROS1 expression by comparing the ROS1-positive population with ROS1-negative tumors. The study was conducted in accordance with the precepts of the Good Clinical Practice guidelines and the Declaration of Helsinki. In addition, the present study was approved by the Ethics Committee of each institute and written informed consent was obtained from all participants.

IHC staining. IHC staining was performed on $4-\mu \mathrm{m}$ sections obtained from formalin-fixed and paraffin-embedded tissue blocks that were subsequantelly mounted on charged slides. Following deparaffinization and rehydration, antigen retrieval was carried out using a Cell Conditioning 1 (CC1) solution for $64 \mathrm{~min}$ at $95^{\circ} \mathrm{C}$. ROS1 IHC assay was performed using three different primary Abs, monoclonal EPMGHR2 (1:200 dilution; Abcam), monoclonal D4D6 (1:50 dilution; Cell Signaling Technology, Inc.) and polyclonal PA1-30318 (1:200 dilution; Thermo Fisher Scientific, Inc.). All assays were carried out using an automated immunostainer (VENTANA. BenchMark ULTRA system; Ventana Medical Systems, Inc.). The intensity of immunostaining in samples was scored by an experienced pathologist using the H-score method. The scoring system was based on intensity ( 0 , no staining; $1+$, weak staining; $2+$, moderate staining; and $3+$, strong staining) and the percentage of positive tumor cells. Therefore, a H-score range of 0-300 was recorded in each case. Based on a previous study in NSCLC (26), a case was considered positive when the $\mathrm{H}$-score was $>100$. In all batches, a negative (without a primary antibody) and a positive (lung adenocarcinoma previously evaluated as ROS1-positive using IHC and FISH analyses) control were employed to evaluate the appropriateness of the IHC analysis.

FISH analysis. In the present study FISH analysis was performed using a commercially available assay (The ZytoLight $^{\circledR}$ SPEC ROS1 Dual Color Break Apart Probe; ZytoVision $\mathrm{GmbH}$ ) according to the manufacturer's recommendations. At least 50 tumor cells from each sample were analyzed and scored according to the guidelines of the European recommendations (27).

The probes labeled the 5' (telomeric) and 3' (centromeric) ends of the fusion breakpoint with green and orange 
fluorochromes, respectively. The criteria for ROS1 FISH interpretation in the tested tumors were the following: i) The break-apart pattern ('conventional' pattern) with one fusion signal and two separated 3 ' and 5' signals; and ii) an atypical pattern showing an isolated 3 ' signal (usually one fusion signal and one isolated $3^{\prime}$ green signal without the corresponding 5 ' signal). The cut-off of rearranged signals to quote ROS1 positivity was based on detection of $\geq 15 \%$ among 50 neoplastic nuclei.

Statistical analysis. No statistical analysis was reported since the expression value of ROS1 with FISH analysis was negative in all cases and the association between ROS1 expression and no associations with clinicopathological parameters of patients was determined.

\section{Results}

Study population. In this study, 150 CAC samples were collected, including 98 surgical specimens and 52 biopsies. The samples were collected from the medical archive corresponding to cases diagnosed between January 2012 and December 2015. The available clinical data were derived from 100 patients' medical records and referring physicians, including 69 males and 31 females with a median age at diagnosis of 70 years (range, 35-84 years). The Eastern Cooperative Oncology Group (ECOG) performance status at diagnosis was 0 in 59, 1 in 27, 2 in 11 patients and unknown in the remaining 3 cases. The primary tumor origin was: intrahepatic bile ducts $(n=56)$; hilar $(n=4)$; extrahepatic $(n=32)$; gallbladder $(n=6)$; while in 2 patients the tumor arised from an unknown primary site. In addition, the clinical stage distribution at diagnosis was as follows: 48 cases exhibited locally recurrent disease; 24 locally advanced disease; 26 metastatic disease; and 2 cases were undetermined. Furthermore, 67 patients underwent surgery and among them, 58 patients experienced radical $\mathrm{R} 0$ resection. At the time of data collection (April 2018), 22 patients were still alive, while 78 died, including 50 who exhibited disease relapse or progression. Patients' baseline characteristics are presented in Table I.

According to the 2015 revised classification of intrahepatic CAC, pathological diagnosis was consistent with conventional small duct type (15 cases), bile ductular type (1 case), intraductal papillary type (1 case), intraductal tubular type (1 case), squamous/adeno squamous cell type (1 case), mucinous/signet ring cell type (5 cases) and undifferentiated type ( 2 cases). Other rare types were reported in 9 cases, whereas in 13 cases the pathological subtype was undetermined. Regarding perihilar and distal CAC, pathological subtype was conventional in 10 cases, intraductal papillary type in 1 case, squamous/adeno squamous cell type in 1 case, mucinous/signet ring cell type in 1 case, while rare and undetermined types were reported in 3 and 12 cases, respectively. Finally, 6 cases of gallbladder adenocarcinoma were included in the study.

Considering treatment strategy, $45 / 48$ patients $(93.75 \%)$ with early stage BTC at diagnosis, received radical surgery as first-line treatment. In 41 of these cases (91\%), no residual tumor (R0) following surgery was observed, while 4 cases exhibited positive surgical margins (R1). Following surgery, 18 patients were treated with gemcitabine-based adjuvant
Table I. Clinical characteristics of patients with biliary tract cancer.

\begin{tabular}{|c|c|}
\hline Parameters & Value, $\mathrm{n}(\%)$ \\
\hline \multicolumn{2}{|l|}{ Sex } \\
\hline Male & $69 / 100(69)$ \\
\hline Female & $31 / 100(31)$ \\
\hline Age, years (median, range) & $70(35-84)$ \\
\hline \multicolumn{2}{|c|}{ Eastern Cooperative Oncology } \\
\hline \multicolumn{2}{|c|}{ Group Performance Status } \\
\hline 0 & $59 / 100(59)$ \\
\hline 1 & $27 / 100(27)$ \\
\hline 2 & $11 / 100(11)$ \\
\hline Unknown & $3 / 100$ \\
\hline \multicolumn{2}{|l|}{ Disease stage } \\
\hline Localized & $48 / 100(48)$ \\
\hline Locally-advanced & $24 / 100(24)$ \\
\hline Metastatic & $26 / 100(26)$ \\
\hline Unknown & $2 / 100(2)$ \\
\hline Surgery & $67 / 100(67)$ \\
\hline $\mathrm{R} 0$ & $58 / 67(87)$ \\
\hline $\mathrm{R} 1$ & $5 / 67 \quad(7)$ \\
\hline $\mathrm{R} 2$ & $2 / 67$ \\
\hline Unknown & $2 / 67$ \\
\hline \multicolumn{2}{|l|}{ Site } \\
\hline Intrahepatic bile ducts & $56 / 100(56)$ \\
\hline Hilar ducts & $4 / 100$ \\
\hline Extrahepatic ducts & $32 / 100(32)$ \\
\hline Gallbladder & $6 / 100$ \\
\hline Unknown & $2 / 100$ \\
\hline
\end{tabular}

CT. At follow-up, 31 patients experienced disease relapse and among them, 21 patients were subsequently treated with alternative platinum- or fluoropyrimidine-based CT treatment.

Furthermore, 24 patients were diagnosed with locally advanced disease, therefore 19 of them underwent surgery and $\mathrm{R} 0$ status was observed in 16 cases. Of the remaining 3 patients, 1 was treated with R1 surgery and 2 patients exhibited R2 positive margins. Additionally, 7/24 locally advanced patients were treated with gemcitabine-based adjuvant $\mathrm{CT}$ after surgery. Conversely, 2 patients received induction CT with platinum/gemcitabine regimen prior to surgery. At follow-up, 12 patients experienced disease relapse with 10 cases being subsequently treated with alternatively platinum- or fluoropyrimidine-based CT. In addition, 26 patients were diagnosed with metastatic disease. Nevertheless, 3 patients received surgical treatment and among them, 1 patient exhibited R0 status.

Overall, 55 patients received a first-line CT for advanced disease (alternatively platinum, gemcitabine- or fluoropyrimidine-based; single agent or combination regimen). Furthermore, following progression, 27 patients were treated with a second-line CT. 
Table II. C-ros oncogene 1 antibodies tested in the cohort of patients with biliary tract cancer.

\begin{tabular}{lcccc}
\hline Primary antibody & Dilution & Commercial source & Epitope & Isotype \\
\hline Monoclonal D4D6 & $1: 50$ & Cell Signaling Technology, Inc. & Carboxy terminal domain & Rabbit IgG \\
Monoclonal EPMGHR2 & $1: 200$ & Abcam & aa 2050-2150 & Rabbit IgG \\
Polyclonal PA1-30318 & $1: 200$ & Thermo Fisher Scientific, Inc. & aa 39-57 & Rabbit IgG \\
\hline
\end{tabular}

aa, amino acid; IgG, immunoglobulin G.

Table III. Distribution of C-ros oncogene 1 expression according to different primary antibody clones used in immunohistochemistry experiments.

\begin{tabular}{lccc}
\hline Sample type & Clone D4D6 & Clone EPMGHR2 & Clone SP384 \\
\hline Biopsy, $\mathrm{n}$ & $0 / 52$ & $7 / 52$ & $5 / 52$ \\
Surgical sample, $\mathrm{n}$ & $0 / 98$ & $21 / 98$ & $17 / 98$ \\
Total, $\mathrm{n}(\%)$ & $0 / 150$ & $28 / 150(18.6)$ & $22 / 150(14.6)$ \\
\hline
\end{tabular}

Table IV. Literature review: C-ros oncogene 1 expression (\%) in cholangiocarcinoma using different antibodies and techniques.

\begin{tabular}{|c|c|c|c|c|c|c|c|c|}
\hline \multirow[b]{2}{*}{ Author, year } & \multicolumn{3}{|c|}{$\mathrm{IHC}$} & \multicolumn{2}{|c|}{ FISH } & \multirow[b]{2}{*}{ RT-qPCR } & \multirow[b]{2}{*}{ Immunoaffinity } & \multirow[b]{2}{*}{ Refs. } \\
\hline & D4D6 & SP384 & EPMGHR2 & Break-apart & Exon 30 & & & \\
\hline Gu et al, 2011 & - & - & - & - & - & - & 8.7 & (20) \\
\hline Liu et al, 2013 & - & - & - & - & - & 0 & - & (21) \\
\hline Graham et al, 2014 & - & - & - & 1 & - & - & - & (22) \\
\hline Arai et al, 2014 & - & - & - & - & - & 0 & - & (28) \\
\hline Lee et $a l, 2015$ & 37.1 & - & - & - & 0 & - & - & (24) \\
\hline Lim et al, 2017 & 19.1 & - & - & 1.1 & - & - & - & $(25)$ \\
\hline
\end{tabular}

IHC, immunohistochemistry; FISH, fluorescence in situ hybridization; RT-qPCR, reverse transcription-quantitative PCR.

ROSI IHC and FISH findings. Although several studies with selected Abs have shown significant correlation between molecular (extractive or in situ) and IHC assays for ROS1 detection, the present study is the first to directly compare various primary Abs against ROS1. Therefore, a group of 150 BTCs was evaluated for ROS1 positivity, using three different primary $\mathrm{Ab}$ clones in order to determine their relative sensitivity and specificity. ROS1 protein expression was evaluated with clone D4D6 and two clones tested for the first time, namely EPMGHR2 and PA1-30318. The main characteristics of the three primary Abs used in the study are summarized in Table II. The scoring distribution of IHC results for each primary Ab is presented in Table III. ROS1 was differentially expressed, based on primary Ab clones used, whereas no ROS1-positive tumors were observed when clone D4D6 was applied. By contrast, $22(14.6 \%)$ and $28(18.6 \%)$ cases showed positive staining when polyclonal PA1-30318 and monoclonal EPMGHR2 Abs were used, respectively. Furthermore, both Abs exhibited higher immunoreactivity in surgically-derived samples compared with that noted to biopsies (Table III and Fig. 1).
Subsequently, FISH analysis was performed in 75 BTC cases, including 25 randomly selected negative cases that were tested with all clones and, 22 and 28 ROS1 positive tumors evaluated with PA1-30318 and EPMGHR2 Abs, respectively. Overall, FISH was negative in all cases. The results obtained with clone D4D6 were consistent with FISH analysis. Therefore, clone D4D6 exhibited the highest specificity and association with FISH assay in detecting ROS1 rearrangements, while false positive results were obtained using the other clones. The results of ROS1 immunostaining reported to date in the literature are summarized in Table IV.

\section{Discussion}

Due to controversial results having previously been reported, ROS1 rearrangements in a large cohort of BTCs were screened. To date, ROS1 rearrangements have been identified only in 6 patients with BTCs $(20,22,25)$. In particular, Gu et al (20) showed the presence of $F I G-R O S 1$ rearrangement in $2 / 23$ patients with CAC (8.7\%), whereas Graham et al (22) reported a single 

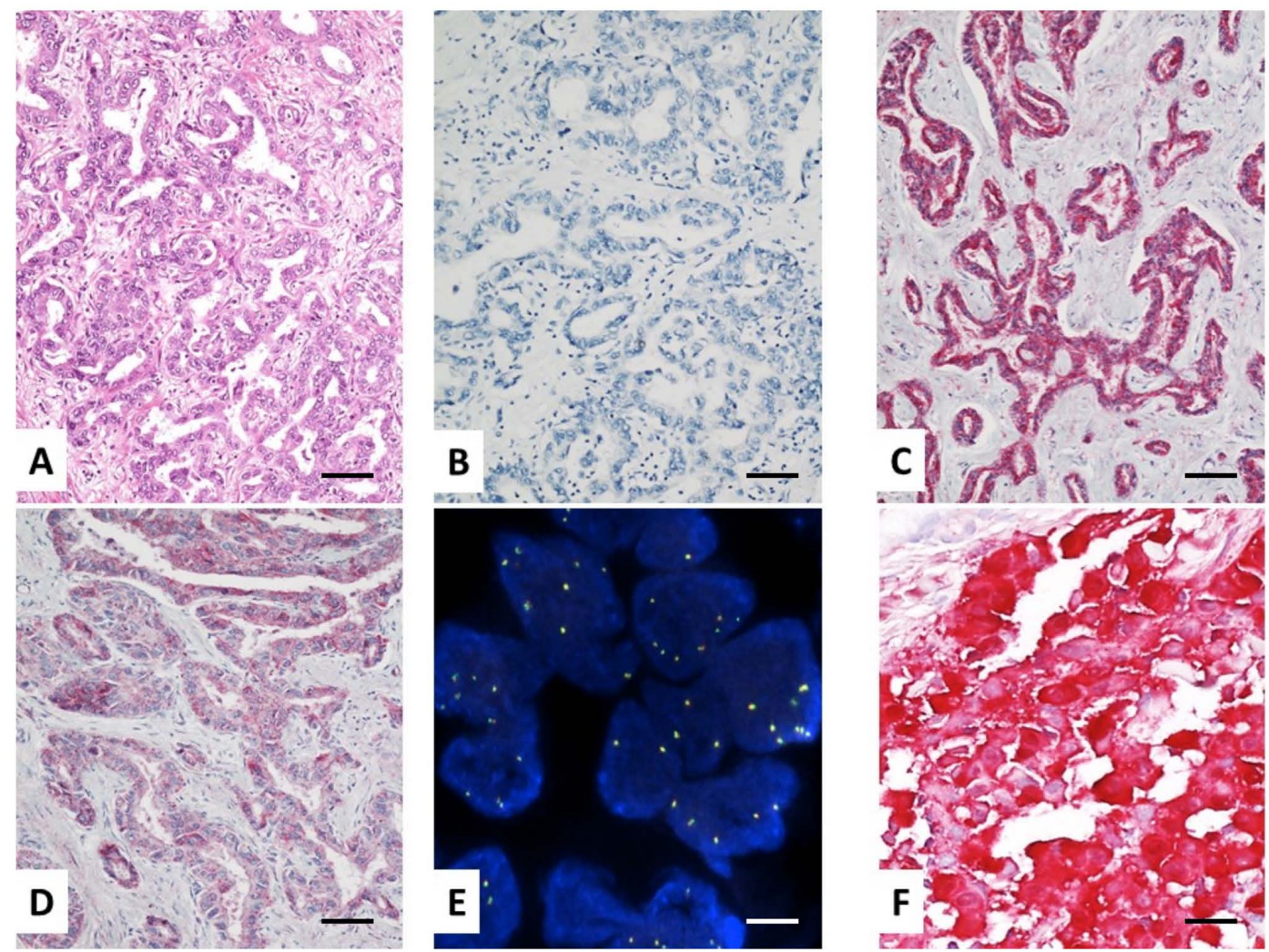

Figure 1. Representative case of intrahepatic CAC. (A) H\&E staining of intrahepatic CAC tissue. (B) IHC assay characterized by negative ROS1 staining using the monoclonal D4D6 Ab. (C) IHC assay characterized by positive ROS1 expression using the polyclonal PA1-30318 and (D) the monoclonal EPMGHR2 Abs. (E) Break-apart fluorescence in situ hybridization probe did not reveal gene rearrangements. Normal fused signals are indicated. (F) A case of ROSI rearrangement detected with the D4D6 Ab in lung adenocarcinoma served as a positive external control in each bath. (A-D and F) Scale bar, $50 \mathrm{~mm}$; (E) Scale bar, 5 mm. CAC, cholangiocarcinoma; H\&E, hematoxylin-eosin; IHC, immunohistochemistry; Ab, antibody; ROS1, c-ros oncogene 1.

case $(1 / 100,1 \%)$ with ROSI translocation and concurrent IDHI mutation. In addition, Lim et al (25) demonstrated a frequency of $R O S 1$ rearrangements of $1.1 \%$ (3/261), in the largest cohort to date. However, the frequency of ROS1 rearrangements has not been fully elucidated. Arai et al (28) and Lim et al (25) revealed that no FIG-ROS1 fusion was detected in CAC when screened by RT-qPCR and FISH, respectively. Screening of ROS1 rearrangements is generally performed using IHC or FISH assays. In lung cancer, the application of both assays is recommended in order to confirm positive results (29).

In particular, we aimed to investigate the incidence of ROSI rearrangements in BTCs by exploiting IHC and FISH techniques. The efficiency of IHC method was examined by comparing three different commercially available primary Abs. To date, only a study conducted by Conde et al (27) compared ROS1 expression using two different ROS1 primary Abs, namely D4D6 and SP384, in a selected cohort of NSCLC patients. This study suggested that SP384 clone was less specific compared with D4D6, as a higher number of false positive results were obtained (25 and 9\% for each Ab, respectively) (27). The results of the present study confirmed that the D4D6 clone was more accurate compared with polyclonal PA1-30318 and monoclonal EPMGHR2 Abs. Furthermore, negative IHC results obtained with the D4D6 Ab, were consistent with the results emerged by FISH analysis. By contrast, the positive results obtained using PA1-30318 (14.6\%) and EPMGHR2 (18.6\%) Abs were not confirmed by FISH analysis. Unlike previous studies, the present study did not detect ROS1 expression in 150 Italian patients with BTCs $(20,22,25,30)$. Previous works by Lee et al $(24)$ and Lim et al (25) have detected ROS1 by IHC with clone D4D6 in 37.1 and $19.1 \%$, respectively. None out of 102 cases (24) and 3 out of 261 cases (1.1\%) (25) were finally resulted as positive at FISH analysis, respectively. Nevertheless, the authors used a very low immunohistochemical cut-off of expression, namely any staining in the study of Lim et al (25) and at least 5\% of stained tumor cells with at least $2+$ of staining intensity in the report by Lee et al (24). In the present study we quoted a positive expression using a more robust cut-off, namely $\mathrm{H}$-score $>100$, then explaining the discrepancy of positive cases between the present and previous observations $(24,25)$.

In a recent study by Lowery et al (31), 195 patients with intrahepatic CAC were prospectively examined by targeted next generation sequencing (NGS) assay, including exons and selected introns from 410 tumor genes. The results of this study demonstrated that the most commonly altered genes in CAC were IDHI (30\%), ARIDIA (23\%), BAPI (20\%), TP53 (20\%) and FGFR2 gene fusions (14\%) (31). In addition, the most common genetic alterations in extrahepatic CAC were detected in KRAS, SMAD4 and STK11 genes. Of note, the authors did not reveal ROS1 gene alterations in their large cohort of patients, although an advanced sequencing technique was performed (31).

In summary, the present study suggests that ROS1 rearrangements in BTCs are not considered reliable molecular targets for the development of novel and selective therapeutic approaches. ROS1 gene alterations in BTCs, that have been 
reported in previous studies, may be considered sporadic cases or false-positive results. Therefore, no further studies are required to detect ROS1 rearrangements in BTCs. However, further research on alternative pathways for the detection of consistent genetic alterations driving to BTC carcinogenesis is needed. These pathways may serve as promising prognostic biomarkers and therapeutic targets for BTCs.

\section{Acknowledgements}

The authors would like to thank Dr Luisa Di Cerbo (Medical Oncology Unit, Azienda Ospedaliero-Universitaria Careggi, Florence, Italy) for her work in data collection and management.

\section{Funding}

The present study was supported by Gruppo Oncologico Italiano di Ricerca Clinica (grant no. GOIRC07/2016) and a grant by Pfizer (grant no. 2017GO-It-FI).

\section{Availability of data and materials}

The datasets used and/or analyzed during the present study are available from the corresponding author on reasonable request.

\section{Authors' contributions}

FM and LA conceived the study design. FM and SP performed manuscript drafting. PP and SP were responsible for data collection and analysis. EV, MP, ACG, FN, AL, CV, EG, GLF, LM, GJ and AB collected data, enrolled patients and performed data interpretation. GR performed data interpretation and wrote the manuscript. LA revised the manuscript. All authors read and approved the final manuscript.

\section{Ethics approval and consent to participate}

The study was conducted in accordance with Good Clinical Practice guidelines, the Declaration of Helsinki, and with approval from each institutional Ethical Committee of the Centers involved (Careggi Hospital-Florence, Regional Hospital Parini-Aosta, Santa Maria delle Croci Hospital-Ravenna, Santa Chiara Hospital-Pisa, Santa Maria Nuova Hospital-Reggio Emilia, IRSST-Meldola, Maggiore Hospital-Parma and San Luca Hospital-Lucca). Written informed consent was obtained from all patients involved.

\section{Patient consent for publication}

Not applicable.

\section{Competing interests}

The authors declare that they have no competing interests.

\section{References}

1. Italian Association Medical Oncology association tumor register The numbers of cancer in Italy, 2017. http://www.registri-tumori it/cms/pubblicazioni/i-numeri-del-cancro-italia-2017. Published September, 2017. Accessed July 6, 2018.
2. Huguet JM, Lobo M, Labrador JM, Boix C, Albert C, Ferrer-Barceló L, Durá AB, Suárez P, Iranzo I, Gil-Raga M, et al: Diagnostic-therapeutic management of bile duct cancer. World J Clin Cases 7: 1732-1752, 2019.

3. Glimelius B, Hoffman K, Sjödén PO, Jacobsson G, Sellström H, Enander LK, Linné T and Svensson C: Chemotherapy improves survival and quality of life in advanced pancreatic and biliary cancer. Ann Oncol 7: 593-600, 1996.

4. Valle J, Wasan H, Palmer DH, Cunningham D, Anthoney A, Maraveyas A, Madhusudan S, Iveson T, Hughes $\mathrm{S}$, Pereira SP, et al; ABC-02 Trial Investigators: Cisplatin plus gemcitabine versus gemcitabine for biliary tract cancer. N Engl J Med 362: 1273-1281, 2010.

5. Jain A and Javle M: Molecular profiling of biliary tract cancer: A target rich disease. J Gastrointest Oncol 7: 797-803, 2016.

6. Jusakul A, Cutcutache I, Yong CH,Lim JQ,Huang MN,Padmanabhan $\mathrm{N}$, Nellore V, Kongpetch S, Ng AWT, Ng LM, et al: Whole-Genome and Epigenomic Landscapes of Etiologically Distinct Subtypes of Cholangiocarcinoma. Cancer Discov 7: 1116-1135, 2017.

7. Javle M,Lowery M, ShroffRT, Weiss KH, Springfeld C, Borad MJ, Ramanathan RK, Goyal L, Sadeghi S, Macarulla T, et al: Phase II Study of BGJ398 in Patients With FGFR-Altered Advanced Cholangiocarcinoma. J Clin Oncol 36: 276-282, 2018.

8. Goyal L, Arkenau HT, Tran B, Soria J-C, Bahleda R, Mak G, Zhu A, Javle M, Hiroshi H, Benedetti F, et al: Early clinical efficacy of TAS-120, a covalently bound FGFR inhibitor, in patients with cholangiocarcinoma. Ann Oncol 28 (Suppl 3): 20, 2017.

9. Blair AB and Murphy A: Immunotherapy as a treatment for biliary tract cancers: A review of approaches with an eye to the future. Curr Probl Cancer 42: 49-58, 2018.

10. Davies KD and Doebele RC: Molecular pathways: ROS1 fusion proteins in cancer. Clin Cancer Res 19: 4040-4045, 2013.

11. Uguen A and De Braekeleer M: ROS1 fusions in cancer: A review. Future Oncol 12: 1911-1928, 2016.

12. Bansal M, He J, Peyton M, Kustagi M, Iyer A, Comb M, White M, Minna JD and Califano A: Elucidating synergistic dependencies in lung adenocarcinoma by proteome-wide signaling-network analysis. PLoS One 14: e0208646, 2019.

13. Bergethon K, Shaw AT, Ou SH, Katayama R, Lovly CM, McDonald NT, Massion PP, Siwak-Tapp C, Gonzalez A, Fang R, et al: ROS1 rearrangements define a unique molecular class of lung cancers. J Clin Oncol 30: 863-870, 2012.

14. Jänne PA and Meyerson M; J2012 PA: ROS1 rearrangements in lung cancer: A new genomic subset of lung adenocarcinoma. J Clin Oncol 30: 878-879, 2012.

15. Shaw AT, Ou SH, Bang YJ, Camidge DR, Solomon BJ, Salgia R, Riely GJ, Varella-Garcia M, Shapiro GI, Costa DB, et al: Crizotinib in ROS1-rearranged non-small-cell lung cancer. N Engl J Med 371: 1963-1971, 2014.

16. Mazières $J$, Zalcman $G$, Crinò L, Biondani $P$, Barlesi F, Filleron $T$, Dingemans AM, Léna H, Monnet I, Rothschild SI, et al: Crizotinib therapy for advanced lung adenocarcinoma and a ROS1 rearrangement: results from the EUROS1 cohort. J Clin Oncol 33: 992-999, 2015.

17. Chiari R, Buttitta F, Iacono D, Bennati C, Metro G, Di Lorito A, Iezzi M, Tiseo M, Mazzoni F, Cappuzzo F, et al: Dramatic response to crizotinib in ROS1 fluorescent in situ hybridizationand immunohistochemistry-positive lung adenocarcinoma: a case series. Clin Lung Cancer 15:470-474, 2014.

18. Landi L, Chiari R, Tiseo M, D'Incà F, Dazzi C, Chella A, Delmonte A, Bonanno L, Giannarelli D, Cortinovis DL, et al: Crizotinib in MET-Deregulated or ROS1-Rearranged Pretreated Non-Small Cell Lung Cancer (METROS): A Phase II, Prospective, Multicenter, Two-Arms Trial. Clin Cancer Res 25: 7312-7319, 2019.

19. Petreni P, Mazzoni F, Meoni G, Lunghi A, Cecere FL, Muto A and Di Costanzo F: Outcome of crizotinib treatment in a young woman with heavily pretreated ROS1-positive lung cancer. Tumori 101: e103-e106,2015.

20. Gu TL, Deng X, Huang F, Tucker M, Crosby K, Rimkunas V, Wang Y, Deng G, Zhu L, Tan Z, et al: Survey of tyrosine kinase signaling reveals ROS kinase fusions in human cholangiocarcinoma. PLoS One 6: e15640, 2011.

21. Liu P, Wu Y, Sun L, Zuo Q and Shi M: ROS kinase fusions are not common in Chinese patients with cholangiocarcinoma. Nan Fang Yi Ke Da Xue Xue Bao 33: 474-478, 2013.

22. Graham RP, Barr Fritcher EG, Pestova E, Schulz J, Sitailo LA, Vasmatzis G, Murphy SJ, McWilliams RR, Hart SN, Halling KC, et al: Fibroblast growth factor receptor 2 translocations in intrahepatic cholangiocarcinoma. Hum Pathol 45: $1630-1638,2014$ 
23. Kipp BR, Voss JS, Kerr SE, Barr Fritcher EG, Graham RP, Zhang L, Highsmith WE, Zhang J, Roberts LR, Gores GJ, et al: Isocitrate dehydrogenase 1 and 2 mutations in cholangiocarcinoma. Hum Pathol 43: 1552-1558, 2012.

24. Lee KH, Lee KB, Kim TY, Han SW, Oh DY, Im SA, Kim TY, Yi NJ, Lee KW, Suh KS, et al: Clinical and pathological significance of ROS1 expression in intrahepatic cholangiocarcinoma. BMC Cancer 15: 721, 2015.

25. Lim SM, Yoo JE, Lim KH, Meng Tai DW, Cho BC and Park YN: Rare Incidence of ROS1 Rearrangement in Cholangiocarcinoma. Cancer Res Treat 49: 185-192, 2017.

26. Rossi G, Jocollé G, Conti A, Tiseo M, Zito Marino F, Donati G, Franco R, Bono F, Barbisan F and Facchinetti F: Detection of ROS1 rearrangement in non-small cell lung cancer: Current and future perspectives. Lung Cancer (Auckl) 8: 45-55, 2017.

27. Conde E,HernandezS,MartinezR,DeCastroJ,Collazo-Lorduy A, Jimenez B, Muriel A, Mate JL, Morán T, Aranda I, et al: Evaluation of a Novel ROS1 Immunohistochemistry Clone (SP384) for the Identification of ROS1 Rearrangements in NSCLC Patients. J Thorac Oncol 13 (Suppl 10): S553-S554, 2018

28. Arai Y, Totoki Y, Hosoda F, Shirota T, Hama N, Nakamura H, Ojima H, Furuta K, Shimada K, Okusaka T, et al: Fibroblast growth factor receptor 2 tyrosine kinase fusions define a unique molecular subtype of cholangiocarcinoma. Hepatology 59: $1427-1434,2014$
29. Bubendorf L, Büttner R, Al-Dayel F, Dietel M, Elmberger G, Kerr K, López-Ríos F, Marchetti A, Öz B, Pauwels P, et al: Testing for ROS1 in non-small cell lung cancer: A review with recommendations. Virchows Arch 469: 489-503, 2016.

30. Abou-Alfa GK, Andersen JB, Chapman W, Choti M, Forbes SJ, Gores GJ, Hong TS, Harding JJ, Vander Heiden MG, Javle M, et al: Advances in cholangiocarcinoma research: Report from the third Cholangiocarcinoma Foundation Annual Conference. J Gastrointest Oncol 7: 819-827, 2016.

31. Lowery MA, Ptashkin R, Jordan E, Berger MF, Zehir A, Capanu M, Kemeny NE, O'Reilly EM, El-Dika I, Jarnagin WR, et al: Comprehensive Molecular Profiling of Intrahepatic and Extrahepatic Cholangiocarcinomas: Potential Targets for Intervention. Clin Cancer Res 24: 4154-4161, 2018. 